\title{
A study of a generalization of bi-ideal, quasi ideal and interior ideal of semigroup
}

\author{
Marapureddy Murali Krishna RaO
}

\begin{abstract}
In this paper, as a further generalization of ideals, we introduce the notion of bi- quasi-interior ideals as a generalization of biideal, quasi ideal, interior ideal, bi-interior ideal and bi-quasi ideal of semigroup and study the properties of bi-quasi-interior ideals of semigroup.
\end{abstract}

\section{INTRODUCTION}

Ideals play an important role in advance studies and uses of algebraic structures. Generalization of ideals in algebraic structures is necessary for further study of algebraic structures. Many mathematicians proved important results and characterizations of algebraic structures by using the concept and the properties of generalization of ideals in algebraic structures. Henriksen [2] and Shabir et al. [15] studied ideals in semirings.

We know that the notion of an one sided ideal of any algebraic structure is a generalization of notion of an ideal. The quasi ideals are generalization of left ideal and right ideal whereas the bi-ideals are generalization of quasi ideals.

In 1952, the concept of bi-ideals was introduced by Good and Hughes [1] for semigroups. The notion of bi-ideals in rings and semigroups were introduced by Lajos and Szasz [8, 9]. Bi-ideal is a special case of $(m-n)$ ideal. In 1956, Steinfeld [16] first introduced the notion of quasi ideals for semigroups and then for rings. Iseki $[5,6,7,8]$ introduced the concept of quasi ideal for a semiring. Quasi ideals bi-ideals in $\Gamma$-semirings studied by Jagtap and Pawar [7]. Murali Krishna Rao [10, 11, 12] introduced the notion of left (right) bi-quasi ideal of semigroup, $\Gamma$-semigroup, $\Gamma$-semiring and study the properties of left bi-quasi ideals.

Murali Krishna Rao [13, 14] studied ideals in ordered $\Gamma$-semirings and introduced the notion of bi-interior ideal as a generalization of quasi ideal,

2010 Mathematics Subject Classification. Primary: 20M17, 06F05.

Key words and phrases. Bi-quasi-interior ideal, bi-interior ideal, bi-quasi ideal, bi-ideal, quasi ideal, interior ideal,regular semigroup, bi-quasi-interior simple semigroup.

Full paper. Received 30 May 2018, revised 5 December 2018, accepted 10 December 2018, available online 15 December 2018. 
bi-ideal and interior ideal of semigroup and study the properties of bi-interior ideals of semigroup, simple semigroup and regular semigroup.

In this paper, as a further generalization of ideals, we introduce the notion of bi- quasi-interior ideal as a generalization of bi-ideal,quasi ideal, interior ideal, bi-interior ideal and bi-quasi ideal of semigroup and study the properties of bi-quasi-interior ideals of semigroup and some characterizations of bi-quasi-interior ideals of semigroup, regular semigroup and simple semigroup.

A necessary and sufficient condition for a semigroup to be regular and simple is proved using bi- quasi-interior ideals semigroup.

\section{Preliminaries}

In this section we will recall some of the fundamental concepts and definitions, which are necessary for this paper.

Definition 2.1. Let $M$ be a semigroup. If there exists $1 \in M$ such that $a \cdot 1=1 \cdot a=a$, for all $a \in M$, is called an unity element of $M$ then $M$ is said to be semigroup with unity.

Definition 2.2. An element $a$ of a semigroup $M$ is called a regular element if there exists an element $b$ of $M$ such that $a=a b a$.

Definition 2.3. A semigroup $M$ is called a regular semigroup if every element of $M$ is a regular element.

Definition 2.4. An element $a$ of a semigroup $M$ is called an idempotent element if $a a=a$.

Definition 2.5. An element $b$ of a semigroup $M$ is called an inverse element of $a$ of $M$ if $a b=b a=1$.

Definition 2.6. A non-empty subset $A$ of $\operatorname{semigroup} M$ is called

(i) a subsemigroup of $M$ if $A A \subseteq A$.

(ii) a left(right) ideal of $M$ if $M A \subseteq A(A M \subseteq A)$.

(iii) an ideal if $M A \subseteq A$ and $A M \subseteq A$.

Definition 2.7. A non-empty subset $A$ of semigroup $M$ is called

(i) an interior ideal of $M$ if $A$ is a subsemigroup of $M$ and $M A M \subseteq A$.

(ii) a quasi ideal of $M$ if $A$ is a subsemigroup of $M$ and $A M \cap M A \subseteq A$.

(iii) a bi-ideal of $M$ if $A$ is a subsemigroup of $M$ and $A M A \subseteq A$.

Definition 2.8. A non-empty subset $B$ of $\operatorname{semigroup} M$ is said to be biinterior ideal of $M$ if $B$ is a subsemigroup of $M$ and $M B M \cap B M B \subseteq B$.

Definition 2.9. Let $M$ be a semigroup. A non-empty subset $L$ of $M$ is said to be left bi-quasi ideal (right bi-quasi ideal) of $M$ if $L$ is a subsemigroup of $M$ and $M L \cap L M L \subseteq L(L M \cap L M L \subseteq L)$. 
Definition 2.10. Let $M$ be a semigroup. $L$ is said to be bi-quasi ideal of $M$ if it is both a left bi-quasi and a right bi-quasi ideal of $M$.

Example 2.1.

(i) Let $Q$ be the set of all rational numbers. Then

$$
M=\left\{\left(\begin{array}{ll}
a & b \\
c & d
\end{array}\right) \mid a, b, c, d \in Q\right\}
$$

is a semigroup with respect to usual matrix multiplication.

(a) If

$$
R=\left\{\left(\begin{array}{cc}
a & b \\
0 & 0
\end{array}\right) \mid 0 \neq a, 0 \neq b \in Q\right\},
$$

then $R$ is a quasi ideal of semigroup $M$ and $R$ is neither a left ideal nor a right ideal.

(b) If

$$
S=\left\{\left(\begin{array}{cc}
a & 0 \\
0 & 0
\end{array}\right) \mid 0 \neq a \in Q\right\},
$$

then $S$ is a bi-ideal of semigroup $M$.

(ii) If

$$
M=\left\{\left(\begin{array}{cc}
a & b \\
0 & c
\end{array}\right) \mid a, b, c \in Q\right\}
$$

and $\Gamma=M$ then $M$ is a semigroup with respect to usual matrix multiplication and

$$
A=\left\{\left(\begin{array}{cc}
a & 0 \\
0 & b
\end{array}\right) \mid 0 \neq a, 0 \neq b \in Q\right\} .
$$

Then $A$ is not a bi-ideal of $\operatorname{semigroup} M$.

\section{BI-QUASI-INTERIOR IDEALS OF SEMIGROUPS}

In this section we introduce the notion of bi-quasi-interior ideal as a generalization of bi-ideal,quasi-ideal and interior ideal of semigroup and study the properties of bi-quasi-interior ideal of semigroup.

Definition 3.1. A non-empty subset $B$ of semigroup $M$ is said to be biquasi-interior ideal of $M$ if $B$ is a subsemigroup of $M$ and $B M B M B \subseteq B$

Every bi-quasi-interior ideal of $\operatorname{semigroup} M$ need not be bi-ideal,quasiideal, interior ideal bi-interior ideal. and bi-quasi ideals of semigroup $M$.

Example 3.1. Let $N$ be a set of all natural numbers. $N$ is a semigroup with respect to usual addition of integers. A subset $I=2 N$ of $N$ is a bi-quasiinterior ideal of $N$ but not bi-ideal,quasi-ideal, interior ideal, bi-interior ideal and bi-quasi ideal of semigroup $N$.

Theorem 3.1. Every bi-ideal of semigroup $M$ is a bi-quasi-interior ideal of semigroupM. 
Proof. Let $B$ be a bi-ideal of semigroup $M$. Then $B M B \subseteq B$.

Therefore $B M B M B \subseteq B M B \subseteq B$.

Hence every bi-ideal of semigroup $M$ is a bi-quasi-interior ideal of $M$.

Theorem 3.2. Every interior ideal of semigroupM is a bi-quasi-interior ideal of $M$.

Proof. Let $I$ be an interior ideal of semigroup $M$.

Then $I M I M I \subseteq M I M \subseteq I$.

Hence $I$ is a bi-quasi-interior ideal of semigroup $M$.

Theorem 3.3. Let $M$ be a semigroup. Every left ideal is a bi-quasi-interior of $M$.

Proof. Let $B$ be a left ideal of semigroup $M$. Then $M B \subseteq B$.

Therefore $B M B M B \subseteq B B M B \subseteq B B B \subseteq B$.

Hence every left ideal of semigroup $M$ is a bi-quasi-interior ideal of $M$.

Corollary 3.1. Let $M$ be a semigroup. Every right ideal is a bi-quasiinterior of $M$.

Corollary 3.2. Let $M$ be a semigroup. Every ideal is a bi-quasi-interior ideal of $M$.

In the following theorems, we mention some important properties and we omit the proofs since proofs are straight forward.

Theorem 3.4. Let $M$ be a semigroup.Every quasi ideal is bi-quasi-interior of $M$.

Theorem 3.5. Let $M$ be a semigroup. Intersection of a right ideal and a left ideal of $M$ is a bi-quasi-interior ideal of $M$.

Theorem 3.6. Let $M$ be a semigroup. If $L$ is a left ideal and $R$ is a right ideal of semigroup $M$ and $B=R L$ then $B$ is a bi-quasi-interior ideal of $M$.

Theorem 3.7. Let $M$ be a semigroup. If $B$ is a bi-quasi-interior ideal and $T$ is a non-empty subset of $M$ such that BT is a subsemigroup of $M$ then $B T$ is a bi-quasi-interior ideal of semigroup $M$.

Theorem 3.8. Let $M$ be a semigroup and $B$ be a subsemigroup of $M$.If $B M M M B \subseteq B$ then $B$ is a bi-quasi-interior ideal of $M$.

Theorem 3.9. Let $M$ be a semigroup and $B$ be a subsemigroup of $M$.If $M M M M B \cap B M M M M \subseteq B$ then $B$ is a bi-quasi-interior ideal of $M$.

Theorem 3.10. Let $M$ be a semigroup and $B$ be a subsemigroup of $M$. $B$ is a bi-quasi-interior ideal of $M$ if and only if there exist a left ideal $L$ and a right ideal $R$ of $M$ such that $R L \subseteq B \subseteq R \cap L$. 
Proof. Suppose $B$ is a bi-quasi-interior ideal of semigroup $M$.

Then $B M B M B \subseteq B$. Let $R=B M B M$ and $L=M B$.

Then $L$ and $R$ are left and right ideals of $M$ respectively. Therefore $R L \subseteq B \subseteq R \cap L$.

Conversely suppose that there exist $L$ and $R$ are left and right ideals of $M$ respectively such that $R L \subseteq B \subseteq R \cap L$. Then

$$
B M B M B \subseteq(R \cap L) M(R \cap L) M(R \cap L) \subseteq R M R M L \subseteq R L \subseteq B .
$$

Hence $B$ is a bi-quasi-interior ideal of semigroup $M$.

Theorem 3.11. The intersection of a bi-quasi-interior ideal $B$ of semigroup $M$ and a right ideal $A$ of $M$ is always bi-quasi-interior ideal of $M$.

Proof. Suppose $C=B \cap A$.

$$
C M C M C \subseteq B M B M B \subseteq B C M C M C \subseteq A M A M A \subseteq A,
$$

since $A$ is a left ideal of $M$, therefore, $C M C M C \subseteq B \cap A=C$.

Hence the intersection of a bi-quasi-interior ideal $B$ of semigroup $M$ and a subsemigroup $A$ of $M$ is always a bi-quasi-interior ideal of $M$.

Theorem 3.12. Let $A$ and $C$ be bi-quasi-interior ideals of semigroup $M$ and $B=A C$. If $C C=C$ then $B$ is a bi-quasi-interior ideal of $M$.

Proof. Let $A$ and $C$ be bi-quasi-interior ideals of semigroup $M$ and $B=A C$ $B B=A C A C=A C C C A C \subseteq A C M C M C \subseteq A C=B$.

Therefore $B=A C$ is a subsemigroup of $M$

$$
B M B M B=A C M A C M A C \subseteq A M A M A C \subseteq A C=B .
$$

Hence $B$ is a bi-quasi-interior ideal of $M$.

Corollary 3.3. Let $A$ and $C$ be bi-quasi-interior ideals of semigroup $M$ and $B=C A$. If $C C=C$ then $B$ is a bi-quasi-interior ideal of $M$.

Theorem 3.13. Let $A$ and $C$ be subsemigroups of $M$ and $B=A C$. If $A$ is the left ideal then $B$ is a bi-quasi-interior ideal of $M$.

Proof. Let $A$ and $C$ be subsemigroups of $M$ and $B=A C$ Suppose $A$ is the left ideal of $M . B B=A C A C \subseteq A C=B$.

$$
B M B M B=A C M A C M A C \subseteq A C=B .
$$

Hence $B$ is a bi-quasi-interior ideal of $M$.

Corollary 3.4. Let $A$ and $C$ be subsemigroups of semigroup $M$ and $B=$ $A C$. If $C$ is a right ideal then $B$ is a bi-quasi-interior ideal of $M$.

Theorem 3.14. Let $M$ be a semigroup and $T$ be a non-empty subset of $M$. Then every subsemigroup of T containing TMTMT is a bi-quasi-interior ideal of semigroup $M$. 
Proof. Let $B$ be a subsemigroup of $T$ containing $T M T M T$. Then

$$
B M B M B \subseteq T M T M T \subseteq B .
$$

Therefore $B M B M B \subseteq B$.

Hence $B$ is a bi-quasi-interior ideal of $M$.

Theorem 3.15. $B$ is a bi-quasi-interior ideal of semigroup $M$ if and only if $B$ is a left ideal of some right ideal of semigroup $M$.

Proof. Suppose $B$ is a left ideal of some right ideal $R$ of semigroup $M$. Then $R B \subseteq B, R M \subseteq R$. Hence $B M B M B \subseteq B M B \subseteq R M B \subseteq R B \subseteq B$. Therefore $B$ is a bi-quasi-interior ideal of semigroup $M$. Conversely suppose that $B$ is a bi-quasi-interior ideal of semigroup $M$. Then $B M B M B \subseteq B$.

Therefore $B$ is a left ideal of right ideal $B M B M$ of $\operatorname{semigroup} M$.

Corollary 3.5. $B$ is a bi-quasi-interior ideal of semigroup $M$ if and only if $B$ is a right ideal of some left ideal of semigroupM.

Theorem 3.16. If $B$ is a bi-quasi-interior ideal of semigroup $M, T$ is a subsemigroup of $M$ and $T \subseteq B$ then $B T$ is a bi-quasi-interior ideal of $M$.

Proof. Suppose $B$ is a bi-quasi-interior ideal of semigroup $M, T$ is a subsemigroup of $M$ and $T \subseteq B$. BTBT $\subseteq B T$.

Hence $B T$ is a subsemigroup of $M$.

We have $M B T M \subseteq M B M$ and $B T M B T \subseteq B M B$, then $B T M B T M B T \subseteq$ $B M B M B T \subseteq B T$.

Hence $B T$ is a bi-quasi-interior ideal of semigroup $M$.

Theorem 3.17. Let $B$ be bi-ideal of semigroup $M$ and $I$ be an interior ideal of $M$. Then $B \cap I$ is a bi-quasi-interior ideal of $M$.

Proof. Obviously $B \cap I$ is subsemigroup of $M$. Suppose $B$ is a bi-ideal of $M$ and $I$ is an interior ideal of $M$. Then

$$
\begin{aligned}
& (B \cap I) M(B \cap I) M(B \cap I) \subseteq B M B M B \subseteq B, \\
& (B \cap I) M(B \cap I) M(B \cap I) \subseteq I M I M I \subseteq I
\end{aligned}
$$

Therefore $(B \cap I) M(B \cap I) M(B I) M \subseteq B \cap I$.

Hence $B \cap I$ is a bi-quasi-interior ideal of $M$.

Theorem 3.18. Let $M$ be a semigroup and $T$ be a subsemigroup of $M$. Then every subsemigroup of $T$ containing TMTMT is a bi-quasi-interior ideal of $M$.

Proof. Let $C$ be a subsemigroup of $T$ containing $T M T M T$. Then

$$
C M C M C \subseteq T M T M T \subseteq C .
$$

Hence $C$ is a bi-quasi-interior ideal of semigroup $M$.

Theorem 3.19. Let $M$ be a semigroup. If $M=M a$, for all $a \in M$, then every bi-quasi-interior ideal of $M$ is a quasi ideal of $M$. 
Proof. Let $B$ be a bi-quasi-interior ideal of a semigroup $M$ and $a \in B$. Then

$$
\begin{gathered}
B M B M B \subseteq B, \\
M a \subseteq M B, \\
M \subseteq M B \subseteq M, \\
M B=M, \\
B M=B M B \subseteq B M B M B, \subseteq B, \\
M B \cap B M \subseteq M \cap B M \subseteq B .
\end{gathered}
$$

Therefore $B$ is a quasi ideal of $M$. Hence the theorem.

Theorem 3.20. The intersection of $\left\{B_{\lambda} \mid \lambda \in A\right\}$ bi-quasi-interior ideals of a semigroup $M$ is a bi-quasi-interior ideal of $M$.

Proof. Let $B=\bigcap_{\lambda \in A} B_{\lambda}$. Then $B$ is a subsemigroup of $M$.

Since $B_{\lambda}$ is a bi-quasi-interior ideal of $M$, we have

$$
\begin{gathered}
B_{\lambda} M B_{\lambda} M B_{\lambda} \subseteq B_{\lambda}, \quad \text { for all } \lambda \in A, \\
\left(\cap B_{\lambda}\right) M\left(\cap B_{\lambda}\right) M\left(\cap B_{\lambda}\right) \subseteq \cap B_{\lambda}, \\
B M B M B \subseteq B .
\end{gathered}
$$

Hence $B$ is a bi-quasi-interior ideal of $M$.

Theorem 3.21. Let $B$ be a bi-quasi-interior ideal of semigroup $M, e \in B$ and $e$ be an idempotent. Then $e B$ is a bi-quasi-interior ideal of $M$.

Proof. Let $B$ be a bi-quasi-interior ideal of semigroup $M$. Suppose $x \in B \cap$ $e M$. Then $x \in B$ and $x=e y, y \in M$.

$$
x=e y=e e y=e(e y)=e x \in e B .
$$

Therefore,

$$
\begin{gathered}
B \cap e M \subseteq e B, \\
e B \subseteq B, \quad e B \subseteq e M, \\
e B \subseteq B \cap e M, \\
e B=B \cap e M .
\end{gathered}
$$

Hence $e B$ is a bi-quasi-interior ideal of $M$.

Corollary 3.6. Let $M$ be a semigroup $M$ and e be an idempotent. Then $e M$ and $M e$ are bi-quasi-interior ideals of $M$.

Theorem 3.22. If $B$ be a left bi-quasi ideal of semigroup $M$, then $B$ is a bi-quasi-interior ideal of $M$.

Proof. Suppose $B$ is a left bi-quasi ideal of semigroup $M$.Then $B M B M B \subseteq$ $M B$ and $B M B M B \subseteq B M B$.

Therefore $B M B M B \subseteq M B \cap M B M \subseteq B$.

Hence $B$ is a bi-quasi-interior ideal of $M$. 
Corollary 3.7. If $B$ be a right bi-quasi ideal of semigroup $M$, then $B$ is a bi-quasi-interior ideal of $M$.

Corollary 3.8. If $B$ be a bi-quasi ideal of semigroup $M$, then $B$ is a biquasi-interior ideal of $M$.

Theorem 3.23. If $B$ be a bi-interior ideal of semigroup $M$, then $B$ is a bi-quasi-interior ideal of $M$.

Proof. Suppose $B$ is a bi-interior ideal of semigroup $M$.Then $B M B M B \subseteq$ $M B M$ and $B M B M B \subseteq M B M$.

Therefore $B M B M B \subseteq M B M \cap M B M \subseteq B$.

Hence $B$ is a bi-quasi-interior ideal of $M$.

We introduce the notion of bi-quasi-interior simple semigroup and characterize the bi-quasi-interior simple semigroup using bi-quasi-interior ideals of semigroup and study the properties of minimal bi-quasi-interior ideals of semigroup..

Definition 3.2. A semigroup $M$ is said to be bi-quasi-interior simple semigroup if $M$ has no bi-quasi-interior ideals other than $M$ itself.

Theorem 3.24. Let $M$ be a simple semigroup. Every bi-quasi-interior ideal is bi-ideal of $M$.

Proof. Let $B$ be a bi-quasi-interior ideal of a simple semigroup $M$.

Then $B M B M B \subseteq B$ and $M B M$ is an ideal of $M$.

Since $M$ is a simple semigroup, we have $M B M=M$. Hence

$$
\begin{aligned}
B M B M B & \subseteq B, \\
B M B & \subseteq B .
\end{aligned}
$$

Hence the theorem is proved.

Theorem 3.25. Let $M$ be a semigroup. Then $M$ is a bi-quasi-interior simple semigroup if and only if $(a)_{b q i}=M$, for all $a \in M$, where $(a)_{b q i}$ is the bi-quasi-interior ideal generated by a.

Proof. Let $M$ be a semigroup. Suppose that $(a)_{b q i}$ is a bi-quasi-interior ideal generated by $a$ and $M$ is a bi-quasi -interior simple semigroup. Then $(a)_{b q i}=M$, for all $a \in M$.

Conversely suppose that $B$ is a bi-quasi-interior ideal of semigroup $M$ and $(a)_{b q i}=M$, for all $a \in M$. Let $b \in B$.

Then $(b)_{b q i} \subseteq B$ implies $M=(b)_{b q i} \subseteq B \subseteq M$.

Therefore $M$ is a bi-quasi-interior simple semigroup.

Theorem 3.26. Let $M$ be a semigroup. $M$ is a bi-quasi-interior simple semigroup if and only if $\langle a\rangle=M$, for all $a \in M$ and where $\langle a\rangle$ is the smallest bi-quasi-interior ideal generated by a. 
Proof. Let $M$ be a semigroup. Suppose $M$ is a bi-quasi-interior simple semigroup, $a \in M$ and $B=M a$.

Then $B$ is a left ideal of $M$.

Therefore, by Theorem [3.5], $B$ is a bi-quasi-interior ideal of $M$.

Therefore $B=M$. Hence $M a=M$, for all $a \in M$.

$$
\begin{aligned}
M a & \subseteq\langle a\rangle \subseteq M, \\
M & \subseteq\langle a\rangle \subseteq M, \\
M & =\langle a\rangle .
\end{aligned}
$$

Suppose $\langle a\rangle$ is the smallest bi-quasi-interior ideal of $M$ generated by $a$, $\langle a\rangle=M, A$ is the bi-quasi-interior ideal and $a \in A$. Then

$$
\begin{gathered}
\langle a\rangle \subseteq A \subseteq M, \\
M \subseteq A \subseteq M .
\end{gathered}
$$

Therefore $A=M$. Hence $M$ is a bi-quasi-interior simple semigroup.

Theorem 3.27. Let $M$ be a semigroup. Then $M$ is a bi-quasi-interior simple $\Gamma$-semigroup if and only if aMaMa $=M$, for all $a \in M$.

Proof. Suppose $M$ is left bi-quasi simple semigroup and $a \in M$.

Therefore $a M a M a$ is a bi-quasi-interior ideal of $M$.

Hence $a M a M a=M$, for all $a \in M$.

Conversely suppose that $a M a M a=M$, for all $a \in M$.

Let $B$ be a bi-quasi-interior ideal of semigroup $M$ and $a \in B$.

$$
M=a M a M a \subseteq B M B M B \subseteq B .
$$

Therefore, $\mathrm{M}=\mathrm{B}$. Hence $M$ is a bi-quasi-interior simple semigroup.

Definition 3.3. A semigroup $M$ is a left (right) simple semigroup if $M$ has no proper left (right) ideal of $M$.

A semigroup $M$ is said to be simple semigroup if $M$ has no proper ideals.

Theorem 3.28. If semigroupM is left simple semigroup then every bi-quasiinterior ideal of $M$ is a right ideal of $M$.

Proof. Let $B$ be a bi-quasi-interior of left simple semigroup. Then $M B$ is a left ideal of $M$ and $M B \subseteq M$. Therefore, $M B=M$. Then $B M=B M B$, and $B M \subseteq B M B M B \subseteq B$, so $B M \subseteq B$.

Hence every bi-quasi-interior ideal is a right ideal of $M$.

Corollary 3.9. If semigroup $M$ is right simple semigroup, then every biquasi-interior ideal of $M$ is a left ideal of $M$.

Corollary 3.10. Every bi-quasi-interior ideal of left and right simple semigroupM is an ideal of $M$.

Theorem 3.29. Let $M$ be a semigroup and $B$ be bi-quasi-interior ideal of $M$. Then $B$ is minimal bi-quasi-interior ideal of $M$ if and only if $B$ is a bi-quasi-interior simple subsemigroup. 
Proof. Let $B$ be a minimal bi-quasi-interior ideal of semigroup $M$ and $C$ be a bi-quasi-interior ideal of $B$. Then $C B C B C \subseteq C$.

Therefore $C B C B C$ is a bi-quasi-interior ideal of $M$.

Since $C$ is a bi-quasi-interior ideal of $B$, then $B=C B C B C \subseteq C$, so $B=C$.

Conversely, suppose that $B$ is a bi-quasi-interior simple subsemigroup of $M$. Let $C$ be a bi-quasi-interior ideal of $M$ and $C \subseteq B$. Then

$$
C=C B C B C \subseteq C M C M C \subseteq B M B M B \subseteq B,
$$

so $B=C$, since $B$ is a bi-quasi-interior simple semigroup.

Hence $B$ is a minimal bi-quasi-interior ideal of $M$.

Definition 3.4. A group $M$ is said to be bi-quasi-interior simple group if $M$ has no bi-quasi-interior ideals other than $M$ itself.

Theorem 3.30. If $M$ is a group then $M$ is bi-quasi-interior simple group.

Proof. Let $B$ be a proper bi-quasi-interior ideal of group $M$ and $1 \neq a \in B$. Since $M$ is a group, there exists $b \in M$ such that $a b=1$. Then $a b x=x=$ $x a b$, for all $x \in M$. Then $x \in B M$. Therefore $M \subseteq B M$. We have $B M \subseteq M$. Hence $M=B M$.

Similarly we can prove $M B=M$. Further, we have

$$
M=M B=B M B M B \subseteq B M \subseteq B .
$$

Therefore, $M=B$.

Hence group $M$ has no proper bi-quasi-interior ideals.

Theorem 3.31. Let $M$ be a semigroup and $B=R L$, where $L$ and $R$ are minimal left ideal and right ideal of $M$ respectively. Then $B$ is a minimal bi-quasi-interior ideal of $M$.

Proof. Obviously $B=R L$ is bi-quasi-interior ideal of $M$. Let $A$ be bi-quasiinterior ideal of $M$ such that $A \subseteq B$.

We have $M A$ is a right ideal. Then

$$
M A \subseteq M B=M R L \subseteq L,
$$

since $L$ is a left ideal of $M$.

Similarly, we can prove $A M \subseteq R$. Therefore $M A=L, A M=R$. Hence $B=A M M A \subseteq A M A M A \subseteq A$. Therefore $A=B$. Hence $B$ is a minimal bi-quasi-interior ideal of $M$.

We characterize regular semigroup using bi-quasi-interior ideals of semigroup.

Theorem 3.32. Let $M$ be a regular semigroup. Then every bi-quasi-interior ideal of $M$ is an ideal of $M$. 
Proof. Let $B$ be a bi-quasi-interior ideal of $M$. Then $B M B M B \subseteq B$, and since $M$ is regular, holds $B M \subseteq B M B$, so $B M \subseteq B M B M B \subseteq B$.

Similarly, we can show that $M B \subseteq B M B M B \subseteq B$.

Hence the theorem is proved.

Theorem 3.33. $M$ is regular semigroup if and only if $A B=A \cap B$ for any right ideal $A$ and left ideal $B$ of semigroup $M$.

Theorem 3.34. Let $M$ be a regular semigroup. Then $B$ is a bi-quasiinterior ideal of $M$ if and only if $B M B M B=B$, for all bi-quasi-interior ideals $B$ of $M$.

Proof. Suppose $M$ is a regular semigroup, $B$ is a bi-interior ideal of $M$ and $x \in B$. Then $B M B M B \subseteq B$ and there exists $y \in M$ such that $x=x y x y x \in$ $B M B M B$. Therefore $x \in B M B M B$. Hence $B M B M B=B$.

Conversely suppose that $B M B M B=B$, for all bi-quasi-interior ideals $B$ of $M$. Let $B=R \cap L$, where $R$ is a right ideal and $L$ is a left ideal of $M$. Then $B$ is a bi-interior ideal of $M$.

Therefore

$$
\begin{aligned}
R \cap L & =(R \cap L) M(R \cap L) M(R \cap L) \\
& \subseteq R M L M L \subseteq R L \subseteq R \cap L,
\end{aligned}
$$

since $R L \subseteq L$ and $R L \subseteq R$.

Therefore $R \cap L=R L$. Hence $M$ is a regular semigroup.

Theorem 3.35. Let $B$ be the bi-quasi-interior ideal of regular semigroup $M$. If $B$ is a bi-quasi-interior ideal of $M$ and $B$ is regular subsemigroup of $M$ then any bi-quasi-interior ideal of $B$ is a bi-quasi-interior ideal of $M$.

Proof. Let $A$ be a bi-quasi-interior ideal of regular subsemigroup $B$ of $M$. Then by Theorem [3.49], $A B A B A=A$. We have $B M B M B=B$ and $A \subseteq A B$. Also,

$$
\begin{gathered}
A M A \Gamma M A \subseteq B M B M B=B, \\
A B A B A=A \subseteq B, \\
A=A B A B A \subseteq A M A \Gamma M A, \\
A M A M A=A .
\end{gathered}
$$

Hence $A$ is a bi-quasi-interior ideal of $M$.

Theorem 3.36. Let $B$ be a subsemigroup of regular semigroup $M$. If $B$ can be represented as $B=R L$, where $R$ is a right ideal and $L$ is a left ideal of $M$ then $B$ is a bi-quasi-interior ideal of $M$.

Proof. Suppose $B=R L$, where $R$ is a right ideal of $M$ and $L$ is a left ideal of $M$. The following holds

$$
B M B M B=R L M R L M R L \subseteq R L=B .
$$


Hence $B$ is a bi-quasi-interior ideal of semigroup $M$.

Conversely suppose that $B$ is a bi-quasi-interior ideal of regular semigroup $M$.

We have $B M B M B=B$. Let $R=B M$ and $L=M B$. Then $R=B M$ is a right ideal of $M$ and $L=M B$ is a left ideal of $M$. The following holds

$$
\begin{gathered}
B M \cap M B \subseteq B M B M B=B, \\
B M \cap M B \subseteq B, \\
R \cap L \subseteq B .
\end{gathered}
$$

We have $B \subseteq B M=R$ and $B \subseteq M B=L$, i.e., $B=R \cap L=R L$, since $M$ is a regular semigroup.

Hence $B$ can be represented as $R L$, where $R$ is the right ideal and $L$ is the left ideal of $M$.

Hence the theorem is proved.

The following theorem is a necessary and sufficient condition for semigroup $M$ to be regular using bi-quasi-interior ideal.

Theorem 3.37. $M$ is a regular semigroup if and only if $B \cap I \cap L \subseteq B I L$, for any bi-quasi-interior ideal $B$, ideal $I$ and left ideal $L$ of $M$.

Proof. Suppose $M$ be a regular semigroup, $B, I$ and $L$ are bi-quasi-interior ideal, ideal and left ideal of $M$ respectively.

Let $a \in B \cap I \cap L$. Then $a \in a M a$, since $M$ is regular, and

$$
a \in a M a \subseteq a M a M a \subseteq B I L .
$$

Hence $B \cap I \cap L \subseteq B I L$.

Conversely suppose that $B \cap I \cap L \subseteq B I L$, for any bi-quasi-interior ideal $B$, ideal $I$ and left ideal $L$ of $M$. Let $R$ be a right ideal and $L$ be a left ideal of $M$. Then by assumption $R \cap L=R \cap M \cap L \subseteq R M L \subseteq R L$. We have $R L \subseteq R, R L \subseteq L$. Therefore $R L \subseteq R \cap L$. Hence $R \cap L=R L$.

Thus $M$ is a regular semigroup.

\section{Conclusion}

As a further generalization of ideals, we introduced the notion of bi-quasiinterior ideal of semigroup as a generalization of ideal, left ideal, right ideal, bi-ideal, quasi ideal and interior ideal of semigroup and studied some of their properties. We introduced the notion of bi-quasi-interior simple semigroup and characterized the bi-quasi-interior simple semigroup, regular semigroup using bi-quasi-interior ideals of semigroup. We proved every bi-quasi ideal of semigroup and bi-interior ideal of semigroup are bi-quasi-interior ideals and studied some of the properties of bi-interior ideals of semigroup. In continuity of this paper, we study prime bi-quasi-interior ideals, prime, maximal and minimal bi-quasi-interior ideals of semigroup. 


\title{
REFERENCES
}

[1] R. A. Good and D. R. Hughes, Associated groups for a semigroup, Bull. Amer. Math. Soc., 58 (1952), 624-625.

[2] M. Henriksen, Ideals in semirings with commutative addition, Amer. Math. Soc. Notices, 5 (1958), 321.

[3] K. Iseki, Quasi-ideals in semirings without zero, Proc. Japan Acad., 34 (1958), 79-84.

[4] K. Iseki, Ideal theory of semiring, Proc. Japan Acad., 32 (1956), 554-559.

[5] K. Iseki, Ideal in semirings, Proc. Japan Acad., 34 (1958), 29-31.

[6] K. Izuka, On the Jacobson radical of a semiring, Tohoku, Math. J., 11 (2) (1959), 409-421.

[7] R. D. Jagatap, Y.S. Pawar, Quasi-ideals and minimal quasi-ideals in O-semirings, Novi Sad J. Math., 39 (2) (2009), 79-87.

[8] S. Lajos, On the bi-ideals in semigroups, Proc. Japan Acad., 45 (1969), 710-712.

[9] S. Lajos, F. A. Szasz, On the bi-ideals in associative ring, Proc. Japan Acad., 46 (1970), 505-507.

[10] M. K. Rao, Left bi-quasi ideals of semirings, Bull. Int. Math. Virtual Inst., 8 (2018), $45-53$.

[11] M. M. K. Rao, Bi-quasi-ideals and fuzzy bi-quasi-ideals of O-semigroups, Bull. Int. Math. Virtual Inst., 7 (2) (2017), 231-242.

[12] M. M. K. Rao, B. Venkateswarlu, N. Rao, Left Bi-quasiideals of O-semirings, Asia Pacific Journal of Mathematics, 4 (2) (2017), 144-153.

[13] M. M. K. Rao, Ideals in ordered $\Gamma$-semirings, Discussiones Mathematicae General Algebra and Applications, 38 (1) (2018), 47-68.

[14] M. M. K. Rao, Bi-interior Ideals in semigroups, Discussiones Mathematicae General Algebra and Applications, 38 (1) (2018), 69-78.

[15] A. M. Shabir, A. Batod, A note on quasi ideal in semirings, Southeast Asian Bull. Math. 7(2004), 923-928.

[16] O. Steinfeld, Uher die quasi ideals, von halbgruppend, Publ. Math., Debrecen, 4 (1956), 262-275.

\author{
Marapureddy Murali Krishna Rao \\ Department of Mathematics \\ GIT, GITAM UNIVERSITY \\ VISAKHAPATNAM - 530045 \\ ANDHRAPRADESH \\ INDIA \\ E-mail address: mmarapureddy@gmail.com
}

